\title{
Patellofemoral Pain Syndrome: An Update
}

\author{
David Hryvniak $\cdot$ Eric Magrum $\cdot$ Robert Wilder
}

Published online: 4 February 2014

(C) Springer Science + Business Media New York 2014

\begin{abstract}
Patellofemoral pain syndrome (PFPS) is one of the most prevalent musculoskeletal conditions seen in sports medicine clinics. The pathophysiology of PFPS is multifactorial. These factors include both extrinsic risk factors, such as changes in training frequency or intensity, training surfaces and inappropriate shoe wear, and intrinsic risk factors, including lower extremity malalignment and muscle and soft tissue imbalances. A combination of biomechanical factors and tissue imbalances causes improper tracking of the patella in the trochlea of the femur, leading to increased stress at the patellofemoral joint. A thorough history and static and dynamic examination can aid the diagnosis of PFPS. Physical therapy continues to be the mainstay of conservative management of patients with PFPS, with a focus on a multimodal treatment approach with an individualized therapy plan based on anatomic and biomechanical factors.
\end{abstract}

Keywords Patellofemoral pain syndrome - Runner's knee $\cdot$ Lower quarter stability $\cdot$ Multimodal treatment

D. Hryvniak $(\bowtie)$

University of Virginia Physical Medicine and Rehabilitation, The Runner's Clinic at UVA, 545 Ray C Hunt Drive, Suite 240, Box 801004, Charlottesville, VA 22908-1004, USA

e-mail:djh3f@virginia.edu

E. Magrum

UVA-Health South Orthopedic Residency Program,

The Runner's Clinic at UVA, Charlottesville, VA, USA

R. Wilder

Chair of Physical Medicine and Rehabilitation at University of Virginia, The Runner's Clinic at UVA, Charlottesville, VA, USA

\section{Introduction}

Patellofemoral pain syndrome (PFPS) is one of the most prevalent musculoskeletal injuries comprising $25 \%$ of knee injuries seen in sports medicine clinics [1,2]. PFPS is the most common knee condition in runners and adolescents [3, 4]. Women are two times more likely to develop PFPS than men [5]. The pathophysiology of PFPS is not fully understood, but is thought to have multiple contributing factors. It is proposed that PFPS arises from a combination of biomechanical factors and tissue imbalances causing improper tracking of the patella in the trochlea of the femur, eventually leading to increased stress at the patellofemoral joint [6]. A thorough history along with a static and dynamic examination can aid in the proper diagnosis of PFPS. Physical therapy continues to be the mainstay of conservative management of patients with PFPS, with emphasis placed on a multimodal treatment approach with an individualized therapy plan based on anatomic and biomechanical factors.

\section{Classification and Risk Factors}

PFPS can be classified based on mechanism of injury, radiographic findings, and biomechanical and alignment factors contributing to the dysfunction and pain. In this classification system, PFPS is broken down into three categories including patellofemoral instability, patellofemoral pain with malalignment and patellofemoral pain without malalignment [7].

Postulated risk factors include both intrinsic and extrinsic risk factors. Extrinsic risk factors include training errors associated with many overuse injuries in sports, such as recent changes in training surface or topography, sudden increase in frequency or intensity, and inappropriate shoe wear. 
Intrinsic risk factors include bony, patellar and lower extremity malalignment along with muscle and soft tissue imbalances. Normally, during knee flexion, the patella follows an S-shaped curve, moving superolaterally through the femoral trochlea with the articular surface of the patella coming into contact with the lateral femoral condyle, and remaining in contact with the femur throughout the flexion arc. The patella is kept in place centrally in the trochlear groove of the femur secondary to the V-shaped anatomy of the patella and the configuration of the femoral condyles. The lateral femoral condyle is typically higher than the medial condyle [8]. Given the high lateral condyle, normally the lateral patellar facet is longer and more sloped to match, with a normal ratio of 3:2 of lateral to medial facet. Bony abnormalities such as dysplasia of the medial or lateral portions of the trochlear groove along with asymmetry of patellar facets can lead to decreased patellar stability and increased risk of PFPS [9, 10].

Lower extremity malalignment can have a significant influence on patellofemoral biomechanics and increase the risk for PFPS. Femoral anteversion, increased quadriceps (Q) angle, patella alta, knee valgus, lateral patellar tilt, laterally displaced tibial tuberosity, abnormal tibial torsion and foot hyperpronation can all lead to malalignment of the patella and lower extremity increasing the risk of PFPS [11, 12]. Increased foot pronation is thought to cause the tibia to internally rotate during the loading phase of gait, thus preventing the tibia from fully externally rotating and the knee from locking during midstance. The femur compensates by rotating internally, allowing the knee to lock, but also increasing the dynamic $Q$ angle and contact pressure between the patella and the lateral trochlear groove, which may increase subchondral bone stress [13].

Muscle and soft tissue imbalances are important risk factors leading to PFPS, but also the most treatable by multimodal therapy. The medial and lateral quadriceps muscles are key dynamic stabilizers of the patellofemoral joint. The vastus medialis obliquus (VMO) is thought to be the main active medial stabilizer of the patella [14]. It has been shown in runners that weakness or delayed activation of the VMO can cause the lateral stabilizers, such as the vastus lateralis (VL), iliotibial band and lateral retinaculum, to overpower the medial stabilizers and increase lateral pressure [15, 16]. Quadriceps muscle tightness can lead to increased contact pressure between the femur and patella [13]. The iliotibial band blends into the lateral retinaculum of the knee; therefore, IT band tightness can lead to lateral patellar tilt and increased pressure between the lateral patella and lateral femoral condyle [11]. Tightness of the hamstring and gastrocnemius musculature can indirectly increase the forces on the patellofemoral joint by applying a constant flexion moment on the patella [17]. Tightness of these muscles also can restrict dorsiflexion at the foot leading to increased pronation at the subtalar joint to compensate [18]. Excessive pronation increases the dynamic $\mathrm{Q}$ angle and therefore lateral contact pressure of the patella [13].

Recently, proximal hip stabilization, specifically strength and activation of the musculature, has been implicated in PFPS. A recent systemic review noted weakness in hip external rotation and abduction in patients with PFPS [19]. Robinson and Nee noted strength deficits in hip extension, while Cichanowski et al. [20, 21] also found weakness in the adductors and internal rotators. Several studies have shown altered patellofemoral joint kinematics in females with PFPS related to excessive medial femoral rotation [19]. Muscle activation may also be an issue as EMG studies have also showed delayed and shorter duration of gluteal muscle activity in individuals with PFPS [22].

\section{Pathophysiology}

The pathophysiology of PFPS is thought to be multifactorial. It is likely that a combination of biomechanical factors along with muscle and soft tissue imbalances lead to improper tracking of the patella in the trochlea of the femur, eventually leading to increased stress at the patellofemoral joint. Thus, microdamage, inflammation and pain of the cartilage and subchondral bone can arise [6]. Patients with PFPS have been found to have increased patellofemoral joint stress, leading to the hypothesis that this in turn leads to increased cartilage and subchondral bone stress, causing damage and pain over time [23, 24]. The lateral retinaculum, synovium, medial patellofemoral ligament and fat pad of Hoffa can also play a role in PFPS [13]. Patellar instability including acute and chronic subluxation or dislocation of the patella can also be a source of PFPS. Other causes of anterior knee pain that must be considered, but occur by different mechanisms, include osteochondral lesions, avulsion fractures, plica, osteoarthritis, patellar and quadriceps tendinitis, bursitis, apophysitis, tendon rupture, patellar fracture, symptomatic bipartite patella, contusion, bone tumor and CRPS [7].

\section{History/Presentation}

PFPS typically presents as anterior knee pain localized behind the kneecap, which can be uni- or bilateral. Onset can be acute or chronic, and may or may not be associated with trauma. The pain typically increases after running, prolonged sitting, squatting, kneeling, and stair climbing or descending [25]. Typically, swelling, locking or instability symptoms are minimal. The perceived instability or 'giving way' that patients may experience is thought to be secondary to pain inhibition limiting full quadriceps contraction. Examination of the knee can help distinguish this 
presentation from a true unstable knee secondary to patellar subluxation, dislocation or ligamentous damage [13].

\section{Physical Examination}

The physical examination for PFPS involves both static and dynamic components. The examination includes standing, sitting, supine and side-lying sections. In the literature, there is a lack of consistency on the biomechanical or alignment differences between patients with PFPS and those without symptoms [26-28]. However, there are static and dynamic risk factors related to patellofemoral malalignment that are thought to predispose patients to developing PFPS. These factors can be isolated during a physical examination and used to design targeted therapy programs [29].

During the standing examination, alignment of the lower leg is assessed by sequentially observing the knees, legs and feet. In particular, the examiner notes evidence of femoral anteversion, genu varum, valgum or recurvatum; external tibial rotation; and foot and ankle alignment while weight bearing. Patellar alignment is also observed. Normally, the patella faces directly forward. A "squinting" or laterally displaced patella is typically abnormal. The Q-angle is a common clinical measurement of the alignment of the lower extremity. The Q-angle is formed by a line connecting the anterior superior iliac spine to the center of the patella and a line connecting the center of the patella to the middle of the anterior tibial tuberosity. It has been reported that a Q-angle greater than $16^{\circ}$ is considered a risk factor for developing PFPS; however, the current literature does not overwhelmingly support this [6, 27].

The standing examination includes a dynamic look at muscular stabilization and lower quarter stability. During a single-leg squat, tracking of the patella is observed in addition to the mechanics of the lower extremity kinetic chain at the hip, knee, ankle and foot. A step-down test has also been shown to be a good screening tool for PFPS. Several studies have shown excessive contralateral pelvic drop, femoral cross adduction and internal rotation, knee abduction, and tibial external rotation and foot hyperpronation to be associated with PFPS [30-32]. These are thought to increase valgus moments on the knee as well as the dynamic Q-angle. It also has been noted that patients with PFPS had greater femoral internal rotation than control subjects using dynamic MRI [33]. The single leg squat tests the stability of the hip in the frontal and transverse planes; therefore, excessive motion in these planes is thought to be secondary to weak hip abductors and external rotators [30, 31, 34, 35].

In the seated position, the knee is palpated for tenderness or effusion. Even a small effusion of 20-30 ml of fluid can inhibit VMO function, affecting proper patellofemoral biomechanics [36]. Tenderness is common, especially over the IT band, VL and lateral retinaculum [37]. Tenderness is also common along the medial patellar facet and, less often, the lateral patellar facet [13]. Patellar position and tracking are assessed. Typically, the patella should face directly forward when the knees are flexed to $90^{\circ}$. Patella alta may be noted if the patella is oriented obliquely toward the ceiling. Patella alta is more common in women and is often found in a congenitally subluxing patella, as it causes the patella to enter the femoral sulcus late in knee flexion. Patella baja is rarely found on examination and occasionally seen as a complication of anterior cruciate ligament reconstruction [13]. Patellar tracking can also be observed by active and passive knee flexion and extension. With normal active extension the patella tracks straight superior or superior to lateral in a 1:1 ratio. A J-sign is an abnormal finding on examination where the patella suddenly deviates laterally at terminal extension as it exits the trochlear groove to create an inverted J-shaped path [25, 38]. Palpation for crepitus is also performed while ranging the knee through flexion and extension.

The supine examination allows for a more detailed examination of leg lengths, lower extremity alignment, patellar tracking, and soft tissue tenderness and flexibility. To assess for leg length discrepancy, the patient performs a bridge, after which leg lengths are measured from the anterior superior iliac spine to the medial malleolus. Discrepancies as small as $1 \mathrm{~cm}$ can have a significant impact on lower extremity biomechanics [39]. The supine examination also allows for the assessment of lower extremity alignment including femoral and tibial torsion. Examination of the patella can be further assessed by special tests including patellar tilt and patellar glide. Patellar tilt is a passive test where the examiner places the thumb on the medial edge of the patella and the index finger on the lateral edge. The examiner then tries to elevate the lateral facet from the lateral condyle. The plane of the patella is compared with the axis of the medial and lateral condyles. The tilt is recorded as positive if the lateral edge of the patella rises above the transcondylar axis. The tilt is negative if below this line. Normally males have tilt of $5^{\circ}$ and females $10^{\circ}$. Lesser degrees of tilt may be associated with a tight retinaculum, which may in turn lead to patellar maltracking or lateral patellar compression syndrome, sometimes requiring surgical release [13]. Patellar glide is assessed with the knee resting in $30^{\circ}$ of flexion. The patella is divided into imaginary quadrants, and then the patella is pushed with the thumb medially and laterally. If the medial glide is less than two quadrants, typically this is secondary to a tight lateral retinaculum. There is an association between patellar hypomobility and a tight ITB [40]. A lateral glide of one quadrant suggests a competent medial restraint. However, a lateral glide of more than three quadrants is consistent with 
an incompetent medial retinaculum. With patellar glide, patellar apprehension should also be noted. Patellar glide is an important indicator of patellar stability; however, it has been shown to overestimate the true amount of lateral displacement as assessed on MRI [41].

In the side-lying position, ITB tightness can be evaluated with Ober's test, and the hip abductors can be tested for strength deficits. The lateral retinaculum can be assessed as well, with the knee flexed at $20^{\circ}$, by passively moving the patella in a medial direction. Normally, the lateral femoral condyle should be exposed with this maneuver [42].

Gait analysis can also be an important dynamic tool for evaluating patients with PFPS, especially in runners. It is thought that impact forces generated during heel-strike and the propulsion phase of running may contribute to an increased risk of developing PFPS [43]. It also has been shown that runners with PFPS have weaker hip abductor muscles, which are associated with an increase in hip adduction during running, especially at the end of a run [44].

\section{Imaging}

Imaging is rarely needed to make the diagnosis of PFPS. It has been shown that patellofemoral malalignment and articular cartilage changes are found in many radiographs and MRI scans of asymptomatic people and typically do not correlate with clinical complaints or clinical outcome after conservative management [45, 46]. Radiographs may be helpful to identify associated pathology such as osteoarthritis, OCD or intra-articular injury. Patellar baja or alta and lateral patellar tilt or subluxation may also be confirmed. Kinematic MRI techniques have been described to provide a detailed evaluation of patellar tracking.

\section{Rehabilitation}

The treatment of PFPS involves a multimodal physical therapy approach including local strengthening, proximal stabilization, lower quarter flexibility, neuromuscular reeducation, orthotics, manual therapy, taping and a variety of other techniques. There are various proposed classification systems to guide conservative management of anterior knee pain. The 2011 International Patellofemoral Research Retreat consensus statement organizes mechanistic influences into three categories: local influences, distal influences and proximal influences on patellofemoral pain to assist with appropriate management based on the current best available evidence and to guide future research $[47 \bullet \bullet]$.

Local factors refer to patellofemoral maltracking dysfunction resulting in overload of the patellar facets, subchondral bone, retinaculum, infra patellar fat pad and other richly innervated synovial-lined tissues in the patellofemoral joint complex.

Patients present with possible congenital anomalies (trochlear dysplasia, patella alta, increased distance of the tibial tubercle-trochlear groove, rotational malalignment) or with soft tissue imbalances creating abnormal pull on the patella with dynamic loading and resultant overload. Treatment should be specific to the direction of patellofemoral maltracking as it is assessed in all planes-medial/ lateral tilt; internal/external rotation; medial/lateral glide; superior/inferior movement. Patellar taping and bracing can address local maltracking factors. In a systematic review investigating PFPS and taping, the authors concluded that patellar taping reduces pain and improves function in people with PFPS during activities of daily living and rehabilitation exercise, but strong evidence to identify the underlying mechanisms is still not available [48]. A recent study quantified the changes in multiplanar patellofemoral kinematics with patellofemoral taping in people with PFPS during dynamic knee movement using fast-phase contrast magnetic resonance imaging. The study concluded that patellar taping shifted the patella inferiorly, reducing symptoms due to an increase in patellofemoral contact area. Taping medialized the patella in participants who demonstrated lateral displacement at baseline and lateralized the patella in participants who demonstrated medial patellar displacement at baseline [49]. This finding reinforces the need to clinically identify the specific alterations in patellofemoral mechanics for patellofemoral taping to be individually utilized as a treatment tool.

Local muscle activation deficits and timing dysfunction between the vastus medialis (VM) and VL are apparent in a subset of PFPS patients. The results of a recent study concluded there is a specific subset of patients with maltracking dysfunction with patellar tilt that correlates with an altered VL:VM activation ratio. They again emphasized accuracy in classification, for specific management of local patellar maltracking with taping to improve VM:VL ratio and resultant pain [50]. A prospective study utilizing military recruits demonstrated that previously healthy individuals with a preexisting alteration of VM/VL EMG onset were more likely to develop PFPS during functional tasks [16]. There has been conflicting evidence regarding taping to improve vastus timing dysfunction, including additional theories to explain the mechanism of pain relief, and functional improvements. Several studies have demonstrated changes in joint position sense and proprioception in PFPS patients [51, 52]. A recent study demonstrated that in a subset of PFPS patients with joint position deficits, patellar taping improved joint position sense and proprioception [53]. In summary, patellofemoral taping in a specific direction to improve the patellofemoral contact area with a resultant decrease in contact forces may be 
beneficial in a specific group of anterior knee pain patients to improve VM/VL activation, position sense and proprioception for improvements in pain and function.

Preferential activation of the VM has been inconclusive through multiple studies. In a recent study, effects of a specific vastus medialis oblique (VMO) retraining program was compared to general quadriceps strengthening on the onset timing of the medial and lateral quadriceps muscle. Following 8 weeks, both groups had similarly improved relative timing of VMO EMG activity in both stair ascent and descent and quadriceps strength gains [54]. This agrees with other reviews of management of PFPS, that general open and closed chain quadriceps strengthening should be an important component of a multimodal approach to management of this patient population [55].

A recent systematic review supports the use of closed kinetic chain strengthening exercises combined with flexibility [56]. Addressing local muscle imbalances includes stretching of specific myofascia evaluated by a flexibility assessment of the lower quarter. Common myofascia to be emphasized in a flexibility/mobility program for PFPS patients include: iliotibial band/tensor fascia latea, quadriceps, especially the rectus femoris; hamstrings, especially the biceps femoris; iliopsoas; and gastrocnemius. Biarticular muscles are more commonly affected in mobility/ flexibility dysfunction. Important techniques include self soft tissue mobilization through the use of a foam roller or other mechanical tool. Static stretching, dynamic stretching/movements, proprioceptive neuromuscular facilitation as well as manual therapy techniques directed at the patellar mobility deficits in all planes are frequently integrated into treatment. Currently, there is insufficient documented evidence to support manual therapy to address local patellofemoral joint dysfunction. However, a randomized controlled trial reported that six sessions of manual therapy, including cross-friction massage stretching directed at the lateral retinaculum and medial patellar glides resulted in improvement in knee flexion and stair climbing [57].

The second proposed classification group is distal influences on lower quarter tracking dysfunction [47••]. In a recent systematic review summarizing the evidence for kinematics associated with PFPS, the authors concluded that individuals with PFPS exhibit delayed peak rear-foot eversion, increased rear-foot eversion at heel strike and a reduction in gait velocity. During running, individuals with PFPS may exhibit increased knee external rotation at the peak knee extension moment, delayed timing of peak rearfoot eversion, increased rear-foot eversion at heel strike, reduced rear-foot eversion excursion during early and total stance, and greater hip adduction [58]. Relationships have been reported among greater rear-foot eversion, coupled tibial internal rotation and femoral adduction with resultant knee dynamic valgus. Thus, treatment interventions focused on addressing the rear-foot may have implications for more proximal dysfunction in PFPS patients [59]. A recent study researching the relationship between a validated static measurement tool (Foot Posture Index) and dynamic movement in PFPS patients reported that a more pronated foot correlated with earlier timing of peak rearfoot eversion [60]. However, static measurements of the foot and ankle may correlate poorly with dynamic movement. A recent study demonstrated increased multiplanar mid-foot mobility compared with asymptomatic controls [61]. Orthotic management to address excessive rear-foot and mid-foot mobility with resultant proximal movement dysfunction is a common treatment modality prescribed for PFPS patients. Evidence exists that prefabricated foot orthoses provide greater short-term improvements in patient-perceived success in individuals with PFPS compared with flat inserts. Prefabricated foot orthoses may reduce transverse plane knee rotation in individuals with PFPS. Combining physiotherapy with prefabricated foot orthoses may be superior to prefabricated foot orthoses used alone [62]. Recent evidence may assist the clinician in assessing which specific PFPS patients may be more appropriate for orthotic management. A clinical prediction rule was derived, identifying that the combination of three of four predictors increased the probability of marked improvement with foot orthoses from 25 to $78 \%$. The four predictors were: (1) poor footwear motion control, (2) less usual pain ( $<22$ on a 100-point visual analog scale), (3) ankle dorsiflexion range of motion $<41^{\circ}$ with the knee flexed and (4) immediate reduction of pain during singleleg squats when wearing orthoses [63]. A clinical reasoning approach with a functional assessment (single leg squat), treatment (orthoses) and re-assessment (improved pain with single leg squat) may be a simple clinical decisionmaking tool to assist with more specific and appropriate prescription of orthoses as a treatment modality in this population. Additional exercises to improve the ability of the foot and ankle complex to control the velocity, timing, and ROM of rear-foot eversion and resultant medial knee collapse is typically incorporated into an exercise prescription when distal factors are a component of the movement dysfunction. Balance and proprioception exercises, utilizing a variety of surfaces, to improve the eccentric pronation of the rear-foot and mid foot in the closed kinetic chain should be a component of the prescribed exercise program.

The third proposed classification group for mechanistic factors for PFPS patients is movement dysfunction caused by proximal influences on lower quarter tracking [47••]. Recent improvements in the ability to assess the patellofemoral joint dynamically through MRI have demonstrated significantly greater lateral patella displacement and 
tilt at various angles of knee flexion. Those studies have concluded that altered patellofemoral joint kinematics in women with PFPS appears to be related to excessive medial femoral rotation as opposed to lateral patella movement and recommend that treatment be directed toward controlling the dysfunctional hip movement pattern causing patellar overload and pain [64••]. There have been fairly consistent findings across studies that PFPS subjects demonstrate altered hip kinematics in the frontal and transverse planes with a variety of functional tasks (walking, running, step down, sit to stand) [58]. Investigators also have been consistent in reporting strength deficits, primarily of the hip external rotators and abductors, in this patient population [65*0]. A recent study, investigating altered hip kinematics in PFPS patients found that peak hip internal rotation and hip adduction were significant predictors of pain, and peak hip adduction was a predictor of function [66]. A systematic review concluded that hip strengthening and coordination should be a primary component of rehabilitation of this patient population [21]. Proximal strengthening should therefore be a key component to management of these patients, primarily trunk strengthening to develop a stable base (pelvis) for larger hip myofascia to effectively generate adequate force to control altered lower quarter alignment and prevent excessive medial knee collapse. Gluteal strengthening should progress from open chain isolation, activation exercises as soon as tolerated to functional closed kinetic chain strengthening emphasizing proper alignment. There is published evidence that provides guidance for EMG assessment of the most effective gluteal medius and maximus strengthening exercises [67]. Timing deficits in gluteal activity also have been evaluated, demonstrating that strengthening in isolation may be inadequate for successful treatment of this population, and neuromuscular re-education training may be required for functional improvements and pain relief. A recent systematic review indicates that delayed and shorter duration of gluteal muscle activity may exist in individuals with PFPS. The authors concluded that rehabilitation targeting these deficits, such as biofeedback or gait retraining, should also be considered in the management of PFPS [24]. In the runner with PFPS, strengthening the proximal trunk and hip myofascia frequently is inadequate to improve the dynamic motor control of the kinematics through the gait cycle. Recent evidence has been expanding on gait retraining to improve kinematic and kinetic variables with a resultant decrease in patellofemoral pain and improved function (running and stair stepping). Various gait retraining tools that are clinically applicable have been evaluated in this population with success, including mirror retraining and 5-10\% step rate or cadence increases. Increasing step rate/cadence by $10 \%$ has been shown to reduce patellofemoral joint-loading forces by $14 \%$ [68]. Clinically, the PFPS patient can adjust their step rate through feedback of a metronome set to the proper higher cadence for running step rate increases and resultant kinetic/kinematic changes that result in decreased patellofemoral forces. Visual and verbal feedback through cues or the use of a mirror to improve the dynamic lower quarter alignment is another effective clinical tool. A recent case series utilizing this treatment modality of visual feedback through the use of a mirror while running reported reduced peak hip adduction, contralateral pelvic drop and hip abduction moment during running. Motor relearning was evident with patients demonstrating skill transfer with kinematic improvements in single leg squatting and step descent. Patients' maintained improvements in pathomechanics one and 3 months post feedback, with concurrent improvements in pain and function [69].

Addressing proximal factors influencing PFPS to establish proximal stability thus includes trunk/core strengthening, hip and gluteal strengthening including external rotators and abductors (progressing from open to closed chain), and importantly neuromuscular control of lower quarter dysfunction in frontal and transverse planes with visual or verbal feedback for improved alignment and loading.

Successful management of PFPS patients involves classification through examination to identify individual local, distal and proximal contributing factors. An individual treatment plan consisting of manual therapy, exercise and feedback/education will target the identified dysfunctions. Despite the fact that physical therapy is the most frequently used conservative treatment for PFPS, studies report that approximately $25 \%$ of patients continue to have pain and dysfunction for more than 1 year after rehabilitation has been completed [70]. A recent study investigated whether impairments of muscle strength, soft tissue length, movement control, postural and biomechanical dysfunction, and psychological factors were associated with improvements in function and pain in PFPS patients. This may be related to psychological factors where fear of pain and movement are a component of their inability to functionally progress [71]. Thus, addressing these psychological factors may be an important part of the overall treatment plan.

\section{Surgery}

Surgery for PFPS should be considered only after failure of conservative care and if the appropriate pathology has been identified. Arthroscopic debridement of the patella or chondroplasty can address articular cartilage injury. A lateral release may be helpful if there is a tight lateral retinaculum and lack of patellar hypermobility. Medial patellofemoral ligament repair may be indicated following 
patellar dislocation, particularly if patellar instability follows. Distal realignment procedures are reserved for those cases of patellar instability associated with an excessive $Q$ angle.

\section{Conclusion}

Physical therapy continues to be the mainstay of conservative management of patients with PFPS. Treatment should consist of a specific multimodal treatment approach, individually designed for the patients' specific pathomechanics that increase loading on the patellofemoral joint and surrounding tissues. Classification through a comprehensive evaluation including a functional biomechanical assessment can assist the treating clinician in prioritizing local, distal and proximal influences on the patellofemoral joint and guide treatment decision making.

\section{Compliance with Ethics Guidelines}

Conflict of Interest D. Hryvniak declares no conflicts of interest. E. Magrum declares no conflicts of interest. R. Wilder declares no conflicts of interest.

Human and Animal Rights and Informed Consent This article does not contain any studies with human or animal subjects performed by any of the authors.

\section{References}

Papers of particular interest, published recently, have been highlighted as:

-• Of major importance

1. Baquie $P$, Brukner P. Injuries presenting to an Australian sports medicine centre: a 12 months study. Clin J Sports Med. 1997;7: $28-31$.

2. Devereaux MD, Lachmann SM. Patello-femoral arthralgia in athletes attending a sports injury clinic. Br J Sports Med. 1984;18:18-21.

3. Baxter M. Knee pain in the paediatric athlete. Paediatric Med. 1986;1:211-8.

4. Taunton JE, Ryan MB, Clement DB, et al. A retrospective casecontrol analysis of 2002 running injuries. Br J Sports Med. 2002;36(2):95-101.

5. Boling M, Padua D, Marshall S, Guskiewicz K, Pyne S, Beutler A. Gender differences in the incidence and prevalence of patellofemoral pain syndrome. Scand J Med Sci Sports. 2009;20(5): 725-30.

6. Earl JE, Vetter CS. Patellofemoral pain. Phys Med Rehabil Clin N Am. 2007;18:439-58.

7. Holmes WS, Clancy WG. Clinical classification of patellofemoral pain and dysfunction. J Orthop Sports Phys Ther. 1998;28:299-306.

8. Goodfellow J, Hungerford DS, Zindel M. Patellofemoral joint mechanics and pathology. 1. Functional anatomy of the patellofemoral joint. J Bone Joint Surg Br. 1976;58:287-90.
9. Hughston JC, Walsh WM, Puddu G. Patellar subluxation and dislocation. Philadelphia: W.B. Saunders; 1984.

10. Walsh WM. Patellofemoral joint. In: DeLee JC, Drez D, editors. Orthopaedic sports medicine, vol. 2. Philadelphia: W.B. Saunders Co.; 1994. p. 1163-248.

11. Fredericson M, Powers CM. Practical management of patellofemoral pain. Clin J Sports Med. 2002;12:36-8.

12. Powers C, Maffucci R, Hampton S. Rearfoot posture in subjects with patellofemoral pain. J Orthop Sports Phys Ther. 1995;22: 155-60.

13. Collado H, Fredericson M. Patellofemoral pain syndrome. Clin Sports Med. 2010;29:370-98.

14. Bose K, Kanagasuntherum R, Osman M. Vastus medialis oblique: an anatomical and physiologic study. Orthopedics. 1980;3: $880-3$.

15. Powers CM. Rehabilitation of patellofemoral joint disorders: a critical review. J Orthop Sports Phys Ther. 1998;28(5):3453-4.

16. Van Tiggelen D, Cowan S, Coorevits P, et al. Delayed vastus medialis obliquus to vastus lateralis onset timing contributes to the development of patellofemoral pain in previously healthy men: a prospective study. Am J Sports Med. 2009;37(6): 1099-105.

17. Sanchis-Alfonso V, Rosello-Sastre E, Martinez-Sanjuan V. Pathogenesis of anterior knee pain syndrome and functional patellofemoral instability in the active young. Am J Knee Surg. 1999;12:29-40.

18. James SL, Jones DC. Biomechanical aspects of distance running injuries. In: Cavanagh PR, editor. Biomechanics of distance running. Champaign: Human Kinetics Books; 1990. p. 249-269.

19. Meira EP, Brumitt J. Influence of the hip on patients with patellofemoral pain syndrome: a systematic review. Sports Health. 2011;3(5):455-65.

20. Robinson R, Nee R. Analysis of hip strength in females seeking physical therapy treatment for unilateral patellofemoral pain syndrome. J Orthop Sports Phys Ther. 2007;37:232-8.

21. Cichanowski H, Schmitt J, Johnson R, Niemuth P. Hip strength in collegiate female athletes with patellofemoral pain. Med Sci Sports Exerc. 2007;39:1227-32.

22. Barton C, Lack S, Malliaras P, Morrissey D. Gluteal muscle activity and patellofemoral pain syndrome: a systematic review. Br J Sports Med. 2013;47(4):1-9.

23. Heino Brechter J, Powers CM. Patellofemoral stress during walking in persons with and without patellofemoral pain. Med Sci Sports Exerc. 2002;34:1582-93.

24. Besier TF, Gold GE, Delp SL, et al. The influence of femoral internal and external rotation on cartilage stresses within the patellofemoral joint. J Orthop Res. 2008;26(12):1627-35.

25. Post WR. Clinical evaluation of patients with patellofemoral disorders. Arthroscopy. 1999;15(8):841-51.

26. Kannus P, Nittymaki S. Which factors predict outcome in the nonoperative treatment of patellofemoral pain syndrome? A prospective follow-up study. Med Sci Sports Exerc. 1994;26(3): 289-96.

27. Messier SP, Davis SE, Curl WW, et al. Etiologic factors associated with patellofemoral pain in runners. Med Sci Sports Exerc. 1991;23(9):1008-15.

28. Thomee R, Renstrom P, Karlsson J, et al. Patellofemoral pain syndrome in young women. I. A clinical analysis of alignment, pain, parameters, common symptoms and functional activity level. Scand J Med Sci Sport. 1995;5(4):237-44.

29. Fredericson M, Yoon K. Physical examination and patellofemoral pain syndrome. Am J Phys Med Rehabil. 2006;85(3):234-43.

30. Ireland $\mathrm{M}$, Willson J, Ballantyne B, et al. Hip strength in females with and without patellofemoral pain. J Orthop Sports Phys Ther. 2003;33:671-6. 
31. Powers C. The influence of altered lower-extremity kinematics on patellofemoral joint dysfunction: a theoretical perspective. J Orthop Sports Phys Ther. 2003;33:639-46.

32. Riegger-Krugh C, Keysor J. Skeletal malalignments of the lower quarter: correlated and compensatory motions and postures. J Orthop Sports Phys Ther. 1996;2:164-70.

33. Powers C, Ward S, Frederiscon M, et al. Patellofemoral kinematics during weight-bearing and non-weight bearing knee extension in persons with lateral subluxation of the patella: a preliminary study. J Orthop Sports Phys Ther. 2003;33(11):677-85.

34. Zeller B, McCrory J, Kibler B, et al. Differences in kinematics and electromyographic activity between men and women during the single-legged squat. Am J Sports Med. 2003;31:449-56.

35. Leetun D, Ireland M, Willson J, et al. Core stability measures as risk factors for lower extremity in athletes. Med Sci Sports Exerc. 2004;36:926-34.

36. de Andrade JR, Grant C, Dixon AS. Joint distention and reflex muscle inhibition in the knee. J Bone Joint Surg Am. 1965;47: 313-22.

37. Merchant AC. Patellofemoral malalignment and instabilities. In: Ewing JW, editor. Articular cartilage and knee joint function: basic science and arthroscopy. New York: Raven Press Ltd.; 1990. p. 79-91.

38. Nissen CW, Cullen MC, Hewett TE, et al. Physical and arthroscopic examination techniques of the patellofemoral joint. J Orthop Sports Phys Ther. 1998;28:277-85.

39. McCaw ST. Leg length inequality. Implications for running injury prevention. Sport Med. 1992;14(6):422-9.

40. Puniello MS. Iliotibial band tightness and medial patellar glide in patients with patellofemoral dysfunction. J Orthop Sports Phys Ther. 1993;17:144-8.

41. Powers CM, Mortenson S, Nishimoto D, et al. Criterion-related validity of a clinical measurement to determine the medial/lateral component of patellar orientation. J Orthop Sports Phys Ther. 1999;29(7):372-7.

42. Grelsamer RP, McConnell J. The patella. Gaithersburg: Aspen; 1998.

43. Thijs Y, De Clercq D, Roosen P, et al. Gait-related intrinsic risk factors for patellofemoral pain in novice recreational runners. $\mathrm{Br}$ J Sports Med. 2008;42(6):466-71.

44. Dierks TA, Manal KT, Hamill J, et al. Proximal and distal influences on hip and knee kinematics in runners with patellofemoral pain during a prolonged run. J Orthop Sports Phys Ther. 2008; 38(8):448-56.

45. Natri A, Kannus P, Jarvinen M. Which factors predict the longterm outcome in chronic patellofemoral pain syndrome? A 7 years prospective follow-up study. Med Sci Sports Exerc. 1998;30(11):1572-7.

46. Haim A, Yaniv M, Dekel S, et al. Patellofemoral pain syndrome: validity of clinical and radiological features. Clin Orthop Relat Res. 2006;451:223-8.

47. •• Powers CM, Bolgla LA, Callaghan MJ, Collins N, Sheehan FT. Patellofemoral pain: proximal, distal, and local factors, 2nd international research retreat. J Orthop Sports Phys Ther. 2012;42(6):A1-A54. The second international research retreat classified anatomical contributing factors that must be identified to create individualized treatment programs.

48. Aminaka N, Gribble PA. A systematic review of the effects of therapeutic taping on patellofemoral pain syndrome. J Athl Train. 2005;40(4):341-51.

49. Derasari A, Brindle TJ, Alter KE, Sheehan FT. McConnell taping shifts the patella inferiorly in patients with patellofemoral pain: a dynamic magnetic resonance imaging study. Phys Ther. 2010; 90(3):411-9.

50. Pal S, Besier TF, Draper CE, Fredericson M, Gold GE, Beaupre GS, Delp SL. Patellar tilt correlates with vastus lateralis: vastus medialis activation ratio in maltracking patellofemoral pain patients. J Orthop Res. 2012;30(6):927-33.

51. Akseki D, Akkaya G, Erduran M, Pinar H. Proprioception of the knee joint in patellofemoral pain syndrome. Acta Orthop Traumatol Turc. 2008;42(5):316-21.

52. Baker V, Bennell K, Stillman B, Cowan S, Crossley KJ. Abnormal knee joint position sense in individuals with patellofemoral pain syndrome. Orthop Res. 2002;20(2):208-14.

53. Callaghan MJ, Selfe J, McHenry A, Oldham JA. Effects of patellar taping on knee joint proprioception in patients with patellofemoral pain syndrome. Man Ther. 2008;13(3):192-9.

54. Bennell K, Duncan M, Cowan S, McConnell J, Hodges P, Crossley K. Effects of vastus medialis oblique retraining versus general quadriceps strengthening on vasti onset. Med Sci Sports Exerc. 2010;42(5):856-64.

55. Rixe JA, Glick JE, Brady J, Olympia RP. A review of the management of patellofemoral pain syndrome. Phys Sportsmed. 2013;41(3):19-28.

56. Harvie D, O'Leary T, Kumar S. A systematic review of randomized controlled trials on exercise parameters in the treatment of patellofemoral pain: what works? J Multidiscip Healthc. 2011;4:383-92.

57. Van der Dolden PA, Roberts DL. Six sessions of manual therapy increase knee flexion and improve activity in people with anterior knee pain: a randomized controlled trial. Austral J Phys Ther. 2006;52:261-4.

58. Barton CJ, Levinger P, Menz HB, Webster KE. Kinematic gait characteristics associated with patellofemoral pain syndrome: a systematic review. Gait Posture. 2009;30(4):405-16.

59. Barton CJ, Levinger P, Crossley KM, Webster KE, Menz HB. The relationship between rearfoot, tibial and hip kinematics in individuals with patellofemoral pain syndrome. Clin Biomech. 2012;27(7):702-5.

60. Barton CJ, Levinger P, Crossley KM, Webster KE, Menz HB. Relationships between the Foot Posture Index and foot kinematics during gait in individuals with and without patellofemoral pain syndrome. J Foot Ankle Res. 2011;4(10):1-7.

61. McPoil TG, Warren M, Vicenzino B, Cornwall MW. Variations in foot posture and mobility between individuals with patellofemoral pain and those in a control group. J Am Podiatr Med Assoc. 2011;101(4):289-96.

62. Barton CJ, Munteanu SE, Menz HB, Crossley KM. The efficacy of foot orthoses in the treatment of individuals with patellofemoral pain syndrome: a systematic review. Sports Med. 2010;40(5):377-95.

63. Barton CJ, Menz HB, Crossley KM. Clinical predictors of foot orthoses efficacy in individuals with patellofemoral pain. Med Sci Sports Exerc. 2011;43(9):1603-10.

64. •- Souza RB, Draper CE, Fredericson M, Powers CM. Femur rotation and patellofemoral joint kinematics: a weight-bearing magnetic resonance imaging analysis. J Orthop Sports Phys Ther. 2010;40(5):277-285. An understanding of the contribution of femoral biomechanics has expanded the rehabilitation approach to include an emphasis on hip stability.

65. •• Lankhorst NE, Bierma-Zeinstra SM, van Middelkoop M. Factors associated with patellofemoral pain syndrome: a systematic review. Br J Sports Med. 2013;47(4):193-206. This systematic review provides a comprehensive listing of factors that must be considered in the evaluation of a patient with patellofemoral syndrome.

66. Nakagawa TH, Serrão FV, Maciel CD, Powers CM. Hip and knee kinematics are associated with pain and self-reported functional status in males and females with patellofemoral pain. Int J Sports Med. 2013;34(11):997-1002.

67. Reiman MP, Bolgla LA, Loudon JK. A literature review of studies evaluating gluteus maximus and gluteus medius activation 
during rehabilitation exercises. Physiother Theory Pract. 2012; 28(4):257-68.

68. Lenhart RL, Thelen DG, Wille CM, Chumanov ES, Heiderscheit $\mathrm{BC}$. Increasing running step rate reduces patellofemoral joint forces. Med Sci Sports Exerc. 2013.

69. Willy RW, Scholz JP, Davis IS. Mirror gait retraining for the treatment of patellofemoral pain in female runners. Clin Biomech. 2012;27(10):1045-51.
70. Piva SR, Fitzgerald GK, Wisniewski S, Delitto A. Predictors of pain and function outcome after rehabilitation in patients with patellofemoral pain syndrome. J Rehabil Med. 2009;41(8):604-12.

71. Piva SR, Fitzgerald GK, Irrgang JJ, Fritz JM, Wisniewski S, McGinty GT, Childs JD, Domenech MA, Jones S, Delitto A. Associates of physical function and pain in patients with patellofemoral pain syndrome. Arch Phys Med Rehabil. 2009;90(2): 285-95. 